\title{
AN INNOVATIVE APPROACH TO TREAT EARLY CHILDHOOD CARIES (ECC) USING SILVER DIAMINE FLOURIDE - A CASE REPORT
}

\author{
Mălina Popa ${ }^{1,2}$, Nicoleta Nikolajevic-Stoican ${ }^{1}$, Simina Boia $^{3}$, Eugen Radu Boia ${ }^{4}$, Magda Mihaela \\ Luca $^{1,2}$
}

\begin{abstract}
Dental decay affects $4-86 \%$ of preschoolers (temporary dentition), and regarding mixed and permanent dentition, the percentage of affected children is between $27-86 \%$. Caries lesions of the temporary teeth is the main dental problem that the preschooler encounters, a problem that affects to some extent the permanent dentition, with repercussions on the development of the stomatognathic system. The use of silver diamine fluoride (SDF), in dental treatment of Early Chilhood Caries (ECC) has been drawing increasing attention cause to its antimicrobial effect on the species Streptococcus Mutans, Actinomyces Naeslundii and Lactobacillus Acidophilus, which are the pathogens most commonly associated with both the onset and the progression of caries, being usually discovered on the surface and in the depth of the lesion. A case report of a 4 years-old female patient suffering of SECC is presented with the application of SDF and glass-ionomer cement restauration showing advantages as carious lesions arrest, improved esthetic appearance avoiding early teeth loss.
\end{abstract}

Keywords: SDF, ECC, SECC, conservative dentistry

\section{Introduction}

In the century of speed, in which dentistry has become less and less invasive and pain control is becoming more effective, our younger patients are still struggling with the fear of dentist and the dental instruments. The desire to alleviate these apprehensions and to eliminate from the treatment the most feared factors for children, namely the rotating instrument, while maintaining a quality therapeutic act, required the replacement of conventional methods of treating carious lesions with less frightening procedures for the pacients.

Dental decay affects $4-86 \%$ of preschoolers (temporary dentition), and regarding mixed and permanent dentition, the percentage of affected children is between $27-86 \%$. [1].
Caries lesions of the temporary teeth is the main dental problem that the preschooler encounters, a problem that affects to some extent the permanent dentition, with repercussions on the development of the stomatognathic system. In this context we note Early Childhood Caries (ECC) but especially Severe Early Childhood Caries (SECC), both types being caused by demineralization of dental structures due to bacterial biofilm [2]. The terms ECC and SECC have been defined by the National Institute of Dental and Craniofacial Research (NIDCR) [3] as:

- It is considered to be ECC when there is one or more carious lesions, with or without the presence of cavities, missing teeth due to caries complications or restorations on the buccal surface of temporary teeth in children up to 6 years of age, or younger. Any rough carious surface present on the teeth in children under 3 years of age is an indicator of SECC;

- $\quad$ SECC refers to children aged 1 to 5 years who have one or more acute or rampant carious lesions, missing teeth due to caries complications or restaorations on the buccal surface.

Silver diamine fluoride (SDF) is a substance based on silver and fluoride that influences the development of carious lesions. The mechanism of action is based on the bactericidal properties of silver that disrupt the cariogenic bacteria and stop the demineralization and degradation of collagen, the process not being fully clarified. Silver-based preparations have a long history in dentistry due to their antimicrobial properties, and its association with fluoride has led to the hypothesis that the solution has the ability to stop the development of carious lesions and also prevent the appearance of new lesions. SDF can also be used in the treatment of dental hypersensitivity [4].

${ }^{1}$ Pediatric Dentistry Research Centre, Pedo-Research, Timişoara, Romania

${ }^{2}$ Discipline of Pediatric Dentistry, Faculty of Dental Medicine, "Victor Babeș" University of Medicine and Pharmacy, Timişoara, Romania

${ }^{3}$ Department of Periodontology, Faculty of Dental Medicine, "Victor Babeș" University of Medicine and Pharmacy, Timișoara, Romania

${ }^{4}$ Department of ENT, Faculty of Medicine, "Victor Babeș" University of Medicine and Pharmacy, Timișoara, Romania E-mail: popa.malina@umft.ro; nicoleta.stoican@umft.ro; simina.boia@umft.ro; eugen.boia@umft.ro; luca.magda@umft.ro 


JURNALUL PEDIATRULUI - Year XXIII,
SDF is a colourless liquid with a pH value around 10,
that has an antimicrobial effect on the species Streptococcus
Mutans, Actinomyces Naeslundii and Lactobacillus
Acidophilus, which are the pathogens most commonly
associated with both the onset and the progression of caries,
being usually discovered on the surface and in the depth of
the lesion. In this sense, fluoride and silver ions contained in
the SDF solution have an inhibitory effect in the formation
of cariogenic biofilms, binding to enzymes that influence
carbohydrate metabolism and sugar absorption, but also
through the three antimicrobial effects of silver: destruction
of the bacterial cell wall, inhibition of enzymatic processes
and replication of bacterial DNA. The most frequently used
SDF concentration was $38 \%$ but there are studies that
mention concentrations of $30 \%$ or $12 \%$, the best results
being obtained with SDF solutions in the highest
concentration. SDF $38 \%$ contains 44,800 ppm fluoride,
being the highest concentration allowed for dental use,
building chemical reactions with hydroxyapatite and forms
the insoluble fluoroapatite and some silver metal
nanoparticles that are attached to hydroxyapatite crystals,
with an inhibitory role on the development of cariogenic
bacteria, which is supposed to result in the ability of SDF to
stop caries without the need to remove the affected structure,
placing the application technique in the class of non-
invasive methods of caries assessment [5].

\section{Case report}

We present the case of an uncooperative 4 years-old female child with Severe Early Childhood Caries. After obtaining informed written consent from the parents, photos of the case were taken. Intra-oral examination revealed the presence of multiple carious lesions on teeth $51,52,61,62$, 74-disto-occlusal lesion, 84-disto-occlusal lesion (Fig. 1). After brushing the teeth, the teeth were isolated with cotton rolls and saliva ejector to ensure a dry working environment, to prevent dilution of the SDF, commercial product Advantage Arrest with a $38 \%$ solution, and to facilitate deep penetration at an optimal concentration. During the 60 seconds in which the SDF solution infiltrates the carious lesion, the patient's attention was distracted with various age-specific jokes and questions to avoid the situation when the child gets bored and becomes agitated or wants to explore the lesion with the tongue, thus compromising the success of treatment.

After the time of action of the material, the carious lesion acquires a black color, being an indicator for the effectiveness of the antimicrobial agent and is given by the silver oxide that binds to the denatured collagen from caries, demineralized tissues or soft tissues (Fig. 2).

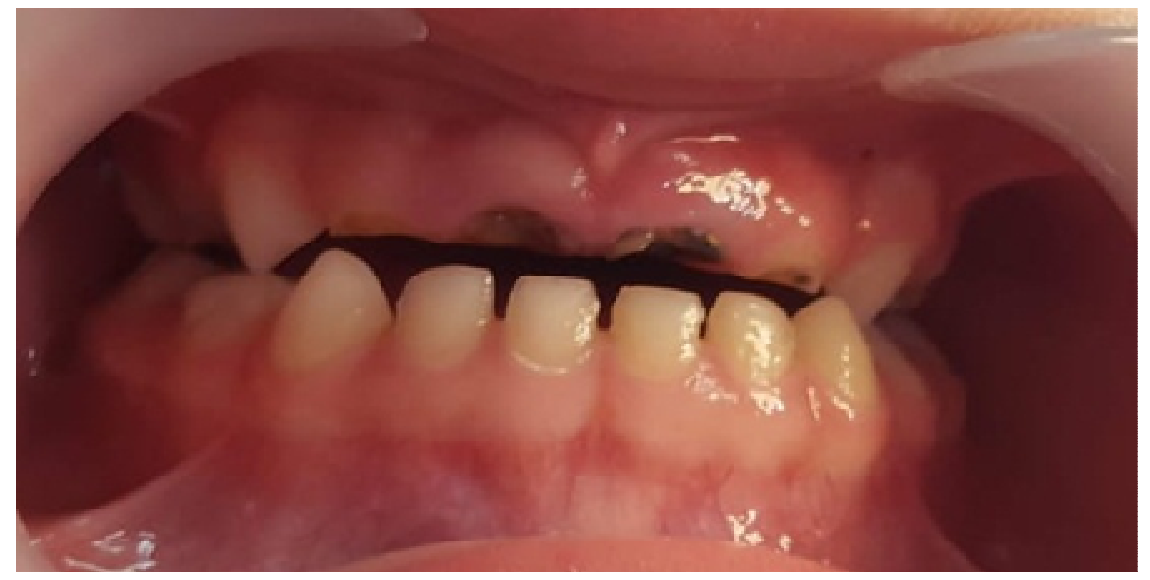

Fig. 1. Intra-oral examination reveals the presence of Sever Early Childhood Caries.
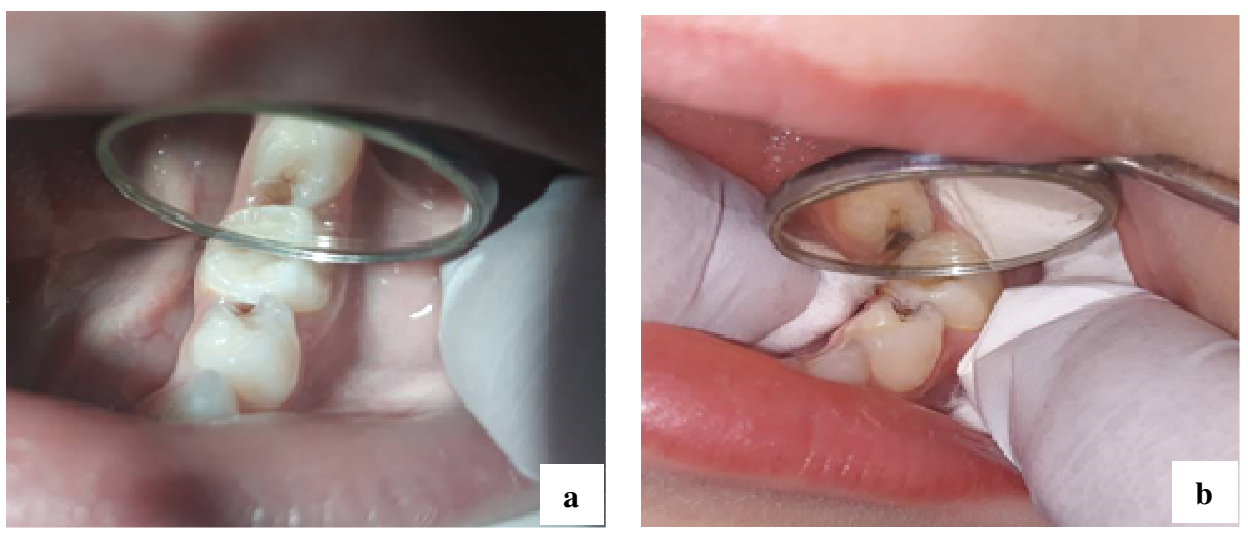

Fig.2 a,b. Initial and final appearance of the disto-occlusal carious lesion on tooth 74. 


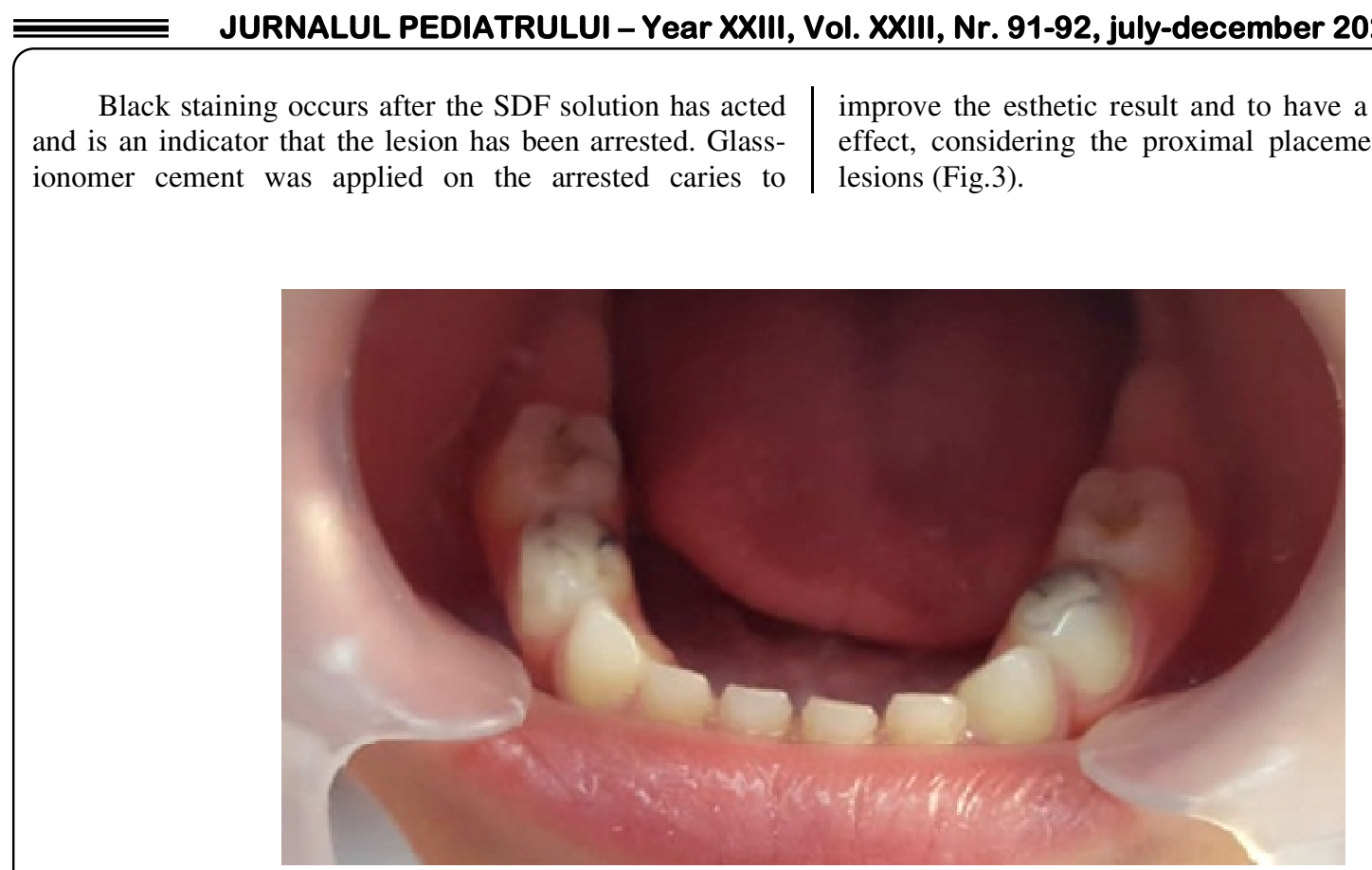

Fig. 3. Final aspect of the arrested and restaurated carious lesions on the mandibular first primary molars.

\section{Discussion}

Arresting carious lesions using SDF solution has a number of advantages given both the simplicity and velocity of clinical procedures, important features that must be taken into account when discussing paediatric patients. A number of features of interest to the medical staff, represented by the elimination of rotary instruments that in the conventional method of treatment can cause pain and anxiety are replaced by a simple application of the SDF liquid. Achieving a complete treatment (treating the carious lesion and applying a final restauration) in such a short time, is a benefit in paedodontic practice because it eliminates all the inconveniences caused by classical treatments with chronophagous stages, rotary handpieces or even with handtools where auxiliary chelators are used in the chemomechanical method [6].

Another advantage is the elimination of difficulties of understanding the commands needed to give to the paediatric patient in the situation of a classic treatment of longer duration, but also the elimination of the stages of preparation, etching, photopolymerization of adhesive systems and photopolymerization of the restauration in layers, all of which require special effort from the patient, and the isolation must be optimal. In comparison, the SDF application is easier, its needed only to isolate the tissues to avoid staining and a soaked in solution micro-brush [7].

SDF treatment eliminates any risk of cross-infection, the $0.1 \mathrm{ml}$ doses being disposable, with no possibility of reconnecting the cap and reusing, and also the other utensils required for the application protocol are disposable (applicators, bibs, saliva ejectors, cotton rolls, single-dose glass-ionomer and even disposable consultation kits) [8].
According to the clinical records from the literature, in cases where the application of SDF is not followed by glassionomer coverage, applications will be continued twice a year until the affected tooth is exfoliated [8].

Another discussion that arises is about the side effects associated with the use of SDF, because the reviews in the literature do not reveal data on long-term use of the solution, blackening of the affected dental areas, currently representing the certain known adverse effect [9].

\section{Conclusion}

The short working time, the comfort of the patient, the simplicity of the technique of use and the complete elimination of the rotary instrumentation, are the clear advantages for which the SDF solution has gained its position among the favorite materials of paediatric dentists. It has shown to be an effective agent in preventing new caries and arresting existing dentine caries.

Compared to other techniques applicable to carious lesions on temporary teeth, arresting lesions with SDF is by far the most advantageous from a financial point of view, the investment being minimal, requiring only the purchase of the product, other special materials not being necessary. Following the application of the SDF solution to arrest carious lesions, the demonstration of the technique and obtaining valid results was a success, and the next step towards evolution will be to improve the aesthetic effect more than is currently done with glass-ionomer cement restauration. 


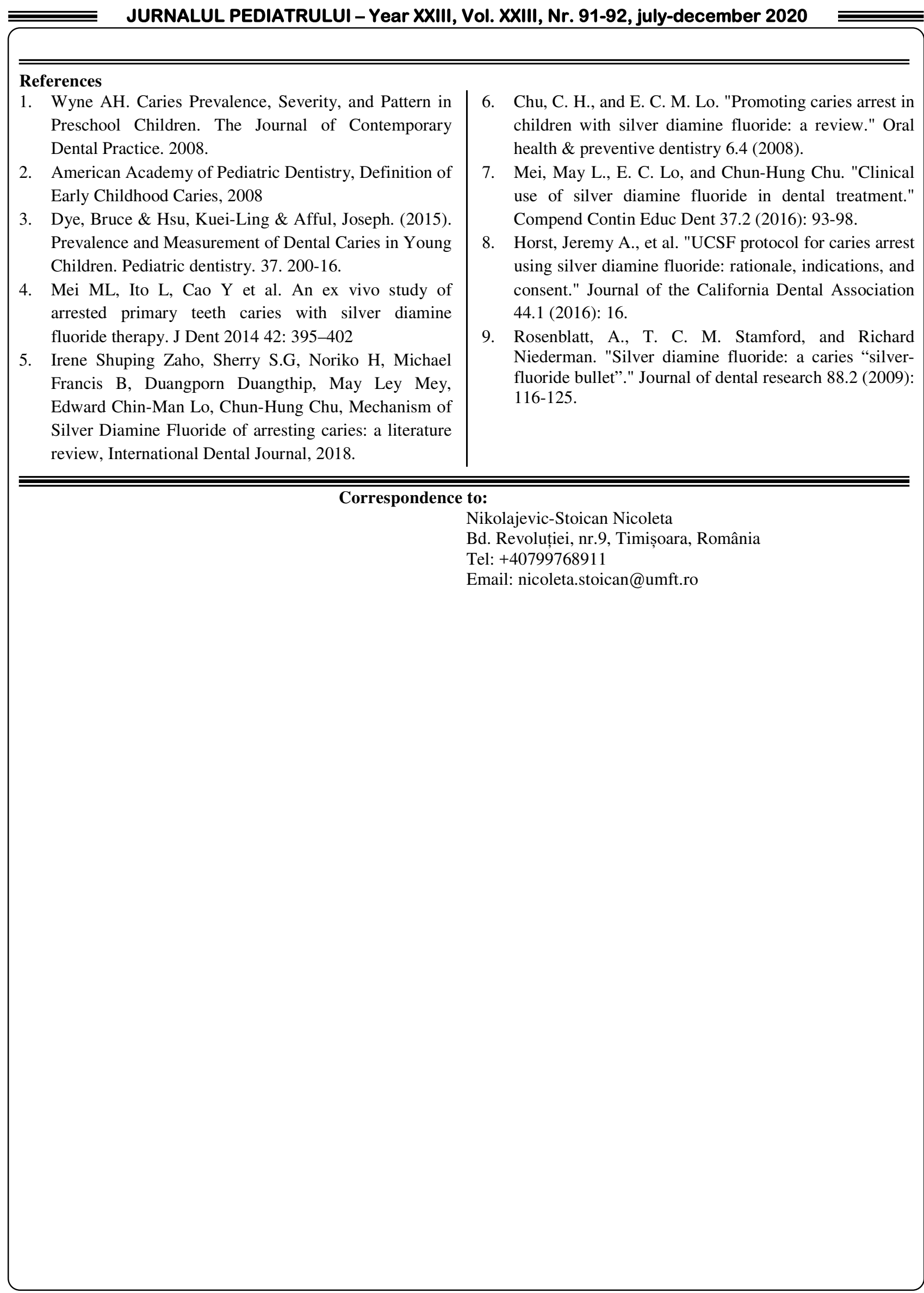

